\title{
A Piezoelectric Force Sensing Glove For Acupuncture Quantification
}

\author{
Kaize $\mathrm{Lin}^{1}$, Jin $\mathrm{CaO}^{2}$, Shuo $\mathrm{Gao}^{1,3, *}$ \\ ${ }^{1}$ School of Instrumentation and Optoelectronic Engineering, Beihang University, Beijing 100191, China. \\ ${ }^{2}$ Department of Psychiatry, Massachusetts General Hospital, Harvard Medical School, 02138, USA \\ ${ }^{3}$ Beijing Advanced Innovation Center for Big Data-Based Precision Medicine, Interdisciplinary Innovation Institute of Medicine \\ and Engineering, Beihang University, Beijing, 100191, China \\ *shuo_gao@buaa.edu.cn
}

\begin{abstract}
Acupuncture is one of the most significant therapies of Chinese traditional medical science, and it is now globally utilized for treatment, e.g. pain management. Traditionally, there is no quantification means for storing masters' skills and examining trainee's learning effect, hence, strongly limiting the development of acupuncture. To address this issue, in this article, a piezoelectric glove based wearable stress sensing system is presented. Experimental results showcase that through the piezoelectric force sensing glove, key parameters (e.g. peak stress at needle) during performing acupuncture are detected and extracted, potentially improving the learning efficiency of trainees and therefore advancing the progress of acupuncture.
\end{abstract}

Keywords-Acupuncture quantification; piezoelectric based glove; normal and shear stress detection

\section{INTRODUCTION}

Acupuncture has been used to treat disorders in China for thousands of years and has been gaining popularity in western countries due to its effectiveness and rarity of adverse effects. Nowadays, via acupuncture, the cure process of nearly 800 illness proves to be effective, including respiratory system diseases, gastrointestinal system diseases, and various nervous system diseases such as epilepsy [1]. In addition, latest researches show that it may do some help to utilize acupuncture as an auxiliary therapy to specific diseases like neurogenic bladder dysfunction (NBD) [2], schizophrenia [3] and cognition impairment [4]. However, without quantitative stress analysis for specific acupuncture process, it is hard to circulate the technique in a large scale due to the opaque manipulation of needle, resulting in a long study period and large variance among doctors. Therefore, in order to solve these problems, it is a necessity to entice modern facilities to acupuncture for quantitative research.

Over the past few decades, the quantification of acupuncture has gained great progress. Advanced information technology used in acupuncture quantification has been reported in [5-6]. However, either the video action capturer or the advanced robotic system focuses on the record and analysis of the manipulating way rather than the stress measurement. Researches on stress measurement system have also been conducted recently. Yang Huayuan [7] has invented an acupuncture manipulation parameters measuring system, which could transform different manipulating actions to electrical

Research funds from the National Natural Science Foundation (grant number 61803017,61827802 ), the "Double FirstClass" university project, and Beihang University Excellent 100 project.

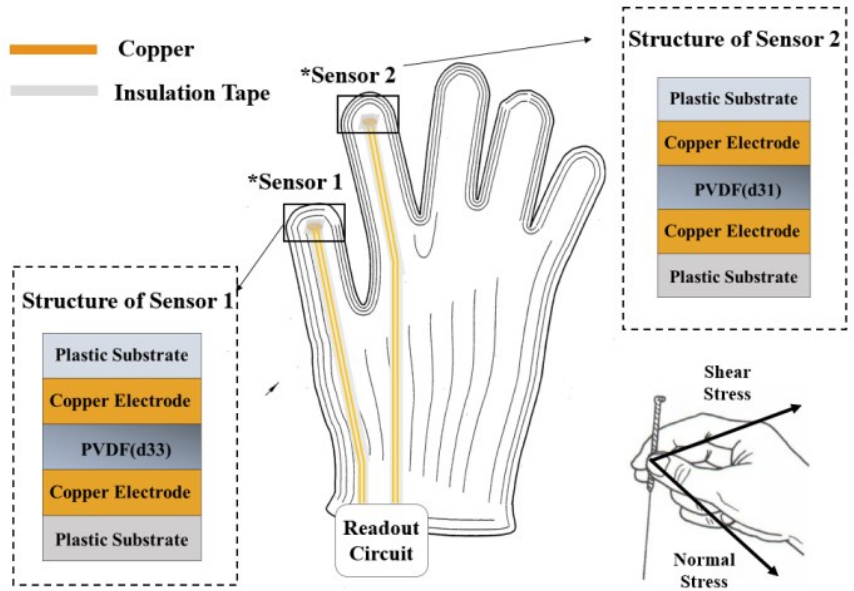

Fig. 1 Conceptual depiction of the in-glove normal and shear force sensing based on piezoelectric architectures.

*Sensor 1 is used to detect normal stress, with a higher piezoelectric coefficient $d_{33}$.

*Sensor 2 is used to detect shear stress, with a higher piezoelectric coefficient $d_{31}$.

signals with four strain gages set around the needle. Nevertheless, the device suffers from some limitations. For instance, the deficiency in shear stress detecting may make the results incomplete, ignoring the important orientation and its parameters. Instead, the piezoelectric material has been attracting large amounts of attention, due to its ability to detect both normal and shear stress as well as its advantages in passive detecting and high accuracy on parameters measuring, potentially allowing for better stress measurement. Furthermore, the small volume of the sensor enables the system to be placed on gloves. Besides, piezoelectric material could present a soft material property and a high tensile resistance.

The stress measuring system, which is attached to the glove's thumb and index finger, is experimentally designed to detect both perpendicular and shear stress. With distinct polarized orientations, PVDF (Polyvinylidene Fluoride) thin films are sensitive to stress in specific directions. Here, $d_{33}$ is used to detect perpendicular stress while $d_{31}$ to detect shear stress. Films with higher $d_{33}$ and with higher $d_{31}$ are placed in thumb and index finger respectively. Considering that the more sensitive 


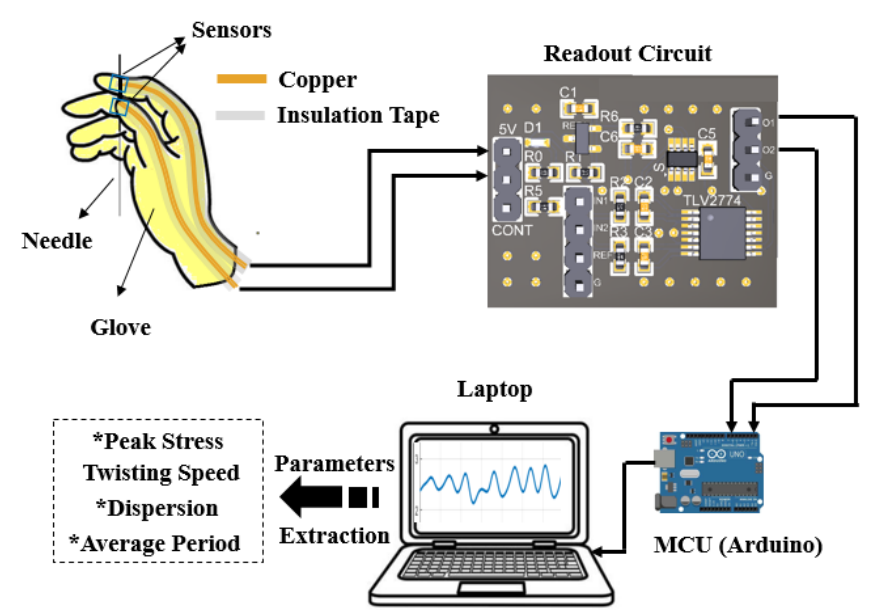

Fig. 2 Architecture of the acupuncture force sensing system.

*Peak Stress refers to the maximum of the stress. *Dispersion refers to the stress dispersion. *Average Period refers to the average lasting time of each stress.

coefficient ( $d_{33}$ in thumb or $d_{31}$ in index finger) is much higher than the lower one, in this case, the lower coefficient could be regarded negligible.

Since there are various kinds of needle manipulation methods, we performed and analyzed the most typical methods (i.e. twisting and lifting-thrusting) in this study. Besides, both manipulating process and force information is indispensable in quantitative analysis for acupuncture. To be specific, parameters including peak stress, twisting speed, dispersion and average period, which could be extracted from normal and shear stress, should be calculated and provided.

To effectively calculate the relative parameters, in this article, a simple technique to analyze the data is developed. Through the technique, each stress is recorded and checked for validity. After the force information and manipulating phases are uploaded to the laptop, the relative parameters can be calculated.

The technique described in this article is validated experimentally with precision and proves to work effectively in various situations. As a result, with perpendicular and shear stress divided and relative parameters calculated, the process is efficaciously quantified.

\section{Methodology}

\section{A. Sensor Fabrication}

Flexible transducers based on piezoelectric materials have been receiving plentiful attention in recent years. It offers a mechanic-electronic conversion, which potentially applies to various fields from wearable electronics to medical instrument [8]. Conventional piezoelectric materials include PVDF [9-10], $\mathrm{ZnO}$ [11], $\mathrm{BaTiO}_{3}$ [12], and PZT (Pb based Lanthanumdoped Zirconate Titanates) [13]. Considering that the manipulation of acupuncture requires a long term needle manipulating as well as rapid response, piezoelectric PVDF-film, which presents both an advanced dynamically mechanic-to-electronic interaction and soft material property, stands out of the crowd.
Data Process

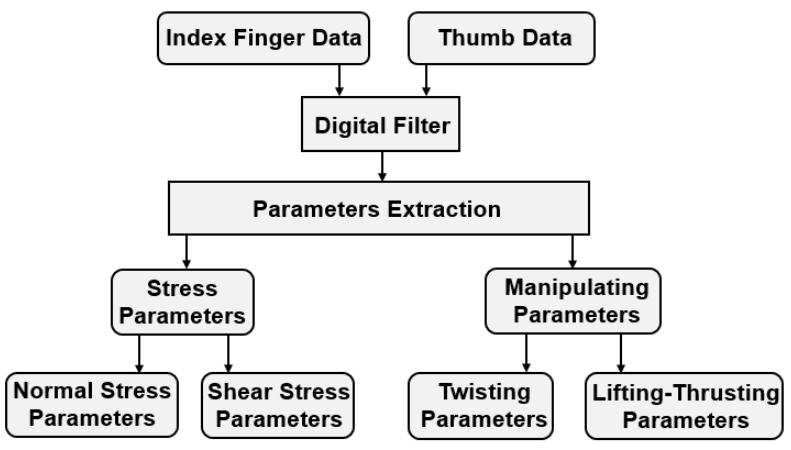

Fig. 3 Flowchart of data process.

The fabrication and the structure of the device are demonstrated in the Fig. 1. The sensor constitutes of 5 layers, with plastic substrate served as a cover and protection. Beneath the plastic layer are the copper electrodes, whose function is to transmit the charges generated by the piezoelectric effect to the readout circuit. In the middle of the sensor is the PVDF film. Served as the sensitive component, PVDF converts the mechanic stress to the production of the charges that are able to transmit through the copper wires stuck in gloves.

\section{B. System Construction}

The piezoelectric-based sensors are placed in gloves, particularly in the edge of thumb and the index finger (or the middle finger, according to the habit of the user). The copper wires are stuck in the internal layer of the glove, connecting the electrodes on one side and the readout circuit on the other. Each sensor has two copper wires, one linking the positive pole of the amplifier (TLV2774, Texas Instruments) and the other linking the ground side. In order to limit the Electro-Magnetic Interference in both the piezoelectric-based sensors and the copper wires, we use the insulation tape to fix them. After the stress signals are amplified by the circuit, the data then is uploaded to the analog-to-digital converter in Arduino Uno.

\section{Algorithm Development}

In order to extract parameters, an algorithm has been designed to help calculate these features. Owing to the generic features of the force, we statistically set a threshold of $2.55 \mathrm{~V}$ of $5 \mathrm{~V}$ for normal stress (2.53 V for shear stress), identifying the valid data. Once the relevant information is recorded, the computer is able to calculate the relevant parameters. Due to the limited space, the algorithm is not presented in the article. The flowchart of data process is presented in Fig. 3.

\section{RESULTS AND DISCUSSION}

The force-time graph of normal and shear stress is depicted in Fig. 4 (a), in which distinct phases of twisting and liftingthrusting are clearly classified and presented. Through monitoring the amplitude of the force, the device has the potential in applying to various new situations. Based on Fig. 4 (a), parameters of normal and shear stress of twisting and liftingthrusting phases can therefore be calculated and extracted. The force-voltage responsivity is experimentally obtained as $0.3 \mathrm{~N} / \mathrm{V}$. Speed as well as dispersion of twisting and lifting-thrusting phases is described in Fig. 4 (b). By using the technique 


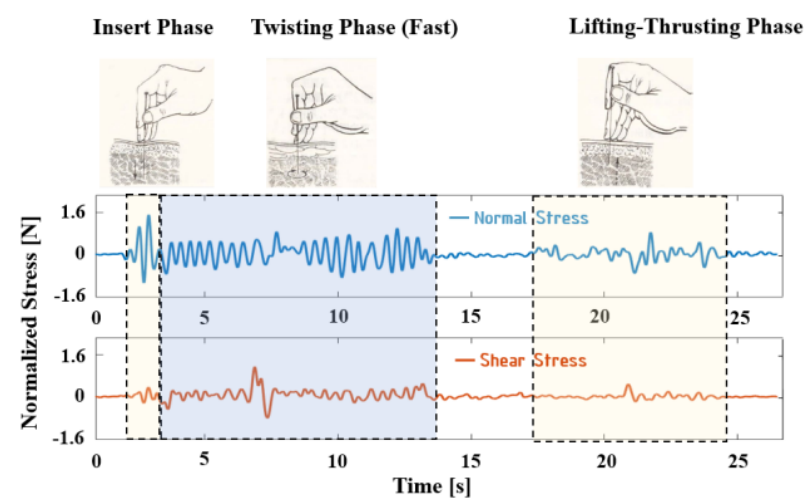

(a)
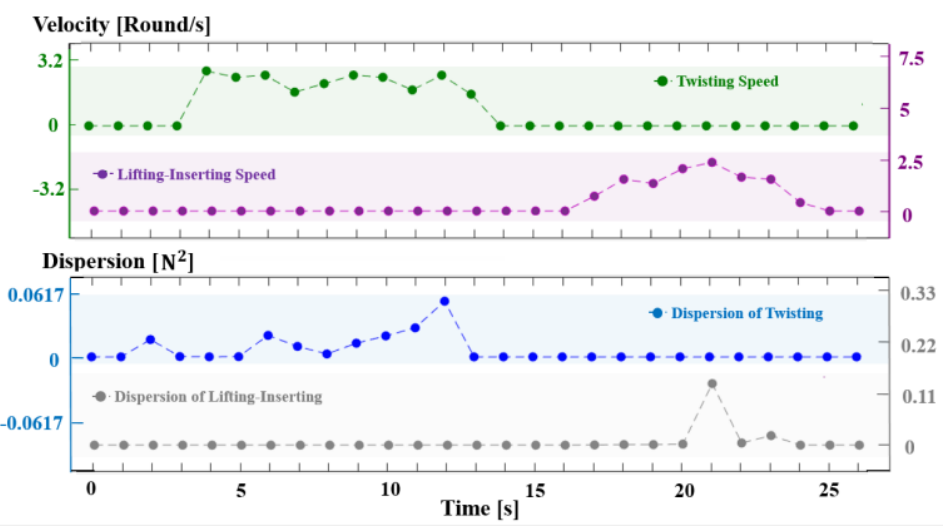

(b)

Fig. 4 (a) Normal and shear stress signals with distinct phases classified. (b) Speed and dispersion of twisting and lifting-thrusting phases to the change of time.

TABLE I SUMMARY OF MAJOR PARAMETERS OF NORMAL AND SHEAR STRESS IN ACUPUNCTURE

\begin{tabular}{|c|c|c|c|}
\hline \multicolumn{3}{|c|}{ System Parameters } \\
\hline \multicolumn{2}{|c|}{ Normal Stress } & \multicolumn{2}{c|}{ Shear Stress } \\
\hline $\begin{array}{c}\text { Number of } \\
\text { Force Sensing }\end{array}$ & 40 & $\begin{array}{c}\text { Number of } \\
\text { Force Sensing }\end{array}$ & 32 \\
\hline Tallest Peak & $1.04 \mathrm{~N}$ & Tallest Peak & $1.19 \mathrm{~N}$ \\
\hline Lowest Peak & $0.22 \mathrm{~N}$ & Lowest Peak & $0.12 \mathrm{~N}$ \\
\hline Average Period & $0.42 \mathrm{~s}$ & Average Period & $0.52 \mathrm{~s}$ \\
\hline Dispersion & $0.05 \mathrm{~N}{ }^{2}$ & Dispersion & $0.04 \mathrm{~N}^{2}$ \\
\hline Average Stress & $0.46 \mathrm{~N}$ & Average Stress & $0.32 \mathrm{~N}$ \\
\hline
\end{tabular}

developed above, relative parameters of normal and shear stress, including peak stress, average period and dispersion, are identified and classified, presented in Table I. More relevant parameters of twisting and lifting-thrusting phases are classified in Table II. By showing the numerous features during acupuncture, the manipulation is effectively quantified and hence can be easily assessed. The variance of the performance during different periods of time mainly owes to the imperfect fabrication of the device and the Electro-Magnetic Interference from the environment.

The device designed in this study is in the purpose of detecting the stress, which is an indispensable factor in acupuncture manipulation. With normal and shear stress divided, distinct phases classified and relative parameters calculated, the experimental results show that the manipulation process of acupuncture is effectively quantified. However, to further study the process of the acupuncture, we need additional sensors like acceleration sensor or angular velocity sensor to get other parameters like lifting-thrusting supplementation and draining [14], which is in our future research plan.

The battery analysis is not presented in the article due to limited space. But considering the small volume of the sensors and the mechanism of stress-electricity conversion, the power consumption can be very low.
TABLE II SUMMARY OF MAJOR PARAMETERS OF TWISTING AND LifTING-Thrusting PHASES IN ACUPUNCTURE

\begin{tabular}{|c|c|c|c|}
\hline \multicolumn{3}{|c|}{ Manipulation Parameters } \\
\hline \multicolumn{2}{|c|}{ Twisting Phase } & \multicolumn{2}{c|}{ Lifting-Thrusting Phase } \\
\hline $\begin{array}{c}\text { Number of } \\
\text { Force Sensing }\end{array}$ & 25 & $\begin{array}{c}\text { Number of } \\
\text { Force Sensing }\end{array}$ & 15 \\
\hline Tallest Peak & $1.27 \mathrm{~N}$ & Tallest Peak & $0.88 \mathrm{~N}$ \\
\hline Lowest Peak & $0.33 \mathrm{~N}$ & Lowest Peak & $0.23 \mathrm{~N}$ \\
\hline Average Period & $0.41 \mathrm{~s}$ & Average Period & $0.43 \mathrm{~s}$ \\
\hline Dispersion & $0.04 \mathrm{~N} 2$ & Dispersion & $0.03 \mathrm{~N} 2$ \\
\hline Average Stress & $0.57 \mathrm{~N}$ & Average Stress & $0.31 \mathrm{~N}$ \\
\hline $\begin{array}{c}\text { Phase Lasting } \\
\text { Time }\end{array}$ & $11.36 \mathrm{~s}$ & $\begin{array}{c}\text { Phase Lasting } \\
\text { Time }\end{array}$ & $7.81 \mathrm{~s}$ \\
\hline Peak Velocity & 2.6 round/s & Peak Velocity & 2.3 round/s \\
\hline
\end{tabular}

\section{NOTES}

Ethics Committee approval has been obtained from the associated clinic. After making an oral and written explanation of the project, informed consent has been gained from relative subjects.

\section{CONCLUSION}

In this article, a piezoelectric-based glove for quantifying force information in acupuncture is presented. Experimental results demonstrate that the presented technique can detect both normal and shear stress while users are twisting the needle. Furthermore, relevant parameters, e.g. speed and peak, which are significant to quantify the process, are successfully extracted. The piezoelectric glove based system developed in this work not only allows trainees to quickly master the acupuncture skills but also provides a means for establishing quantification standards, hence enhancing the development of acupuncture. 


\section{REFERENCES}

[1] X. Xue, Y. Huan, Q. Liu, "Summary of the Mechanism of Acupuncture and Moxibustion in the Treatment of Epilepsy" in Journal of Liaoning University of Traditional Chinese J. Clerk Maxwell, A Treatise on Electricity and Magnetism, 3rd ed., vol. 20, no. 1, pp. 123-125, 2018.

[2] H. Lei, Y. Fu, G. Xu, Z. Yin, L. Zhao, and F. J. M. Liang, "Different types of acupuncture and moxibustion therapy for neurogenic bladder after spinal cord injury: A systematic review and network meta-analysis study protocol," Medicine, vol. 99, no. 1, p. e18558, 2020.

[3] M. Li, "Research of acupuncture and moxibustion therapy of schizophrenia", in Hunan Journal of Traditional Chinese Medicine, 2015:284-288. R. Nicole, "Title of paper with only first word capitalized, " J. Name Stand. Abbrev., in press. no. 9, pp. 194-197, 2017.

[4] E. Lin, D. Lin. "Literature research on channels and acupoints of acupuncture therapy in treating cognitive dysfunction", China Journal of Traditional Chinese Medicine and Pharmacy, vol. 31, no. 11, pp. 48354837, 2016.

[5] P. Yang, X. Sun, Y. Ma, C. Zhang, W. Zhang. "Quantification research on acupuncture manipulation based on video motion capture", Journal of Medical Biomechanics, vol. 31, no. 2, pp. 154-159, 2016.

[6] M. Ma. "Design and implementation of advanced acupuncture quantitative robot system",Tianjing University, 2018.

[7] T. Liu, H. Yang, X Gu. "Development of ATP-I Acupuncture Manipulator Parameter Detector", Chinese Acupuncture \& Moxibustion, 2003.
[8] M. S. Vijaya, "Piezoelectric materials and devices: applications in engineering and medical sciences". CRC Press, 2016.

[9] S. Gao, X. Wu, H. Ma, J. Robertson and A. Nathan. "Ultrathin multifunctional graphene-PVDF layers for multidimensional touch interactivity for flexible displays". ACS applied materials \& interfaces, 9(22), pp.18410-18416, 2017.

[10] S. Gao, C. Huang and L. Wu. "Piezoelectric material based technique for concurrent force sensing and energy harvesting for interactive displays". In 2017 IEEE SENSORS (pp. 1-3). IEEE, October, 2017.

[11] Y. K. Mishra and R Adelung. "ZnO tetrapod materials for functional applications". Materials Today, 21(6), pp.631-651, 2018.

[12] J. Yan and Y. G. Jeong. "High performance flexible piezoelectric nanogenerators based on $\mathrm{BaTiO} 3$ nanofibers in different alignment modes". ACS applied materials \& interfaces, 8(24), pp.15700-15709, 2016.

[13] M. S. Noh, S. Kim, D. K. Hwang, and C. Y. Kang. "Self-powered flexible touch sensors based on PZT thin films using laser lift-off". Sensors and Actuators A: Physical, 261, pp.288-294, 2017.

[14] G. Xu, H. Yang, T. Liu, M. Gao. "Research Status and Progress on Acupuncture Technique Parameter Quantitation". Chinese Archives of Traditional Chinese Medicine, 2017. 